Herausgeber:

Prof. Dr. Klaus Boers, Universität Münster | Prof. Dr. Heinz Cornel, Alice Salomon-FH Berlin | Prof. Dr. Frieder Dünkel, Universität Greifswald | Prof. Dr. Monika Frommel, Universität Kiel | Prof. Dr. Wolfgang Heinz, Universität Konstanz | Prof. Gabriele Kawamura-Reindl, Georg-SimonOhm-FH Nürnberg | Prof. Dr. Joachim Kersten, Deutsche Hochschule der Polizei, Münster | Prof. Dr. Karl-Ludwig Kunz, Institut für Strafrecht und Kriminologie, Bern | Prof. Dr. Heribert Ostendorf, Forschungsstelle für Jugendstrafrecht und Kriminalprävention an der Universität Kiel | Prof. Dr. Bernd-R. Sonnen, Universität Hamburg | PD Dr. Wolfgang Stangl, Institut für Rechts- und Kriminalsoziologie, Wien | Dr. Joachim Walter, ehemals JVA Adelsheim

\title{
Die Mehrzahl der Prostituierten sind keine Opfer, wieso tut sich die Gesetzgebung so schwer, diese Branche angemessen zu regulieren?
}

Ina Hunecke

Rückblickend erscheint die Geschichte der Kriminalisierung und Legalisierung der Prostitution in sich wiederholenden Kreisen zu verlaufen. Bereits im Jahre 1985 tagte ein nationaler Prostituiertenkongress in Berlin. Damals war das Thema noch neu und forderte die Anerkennung dieses Berufes, Zugang zum Sozialversicherungssystem und die Abschaffung der vom Reichsgericht konstruierten, erst 2002 mit dem ProstG abgeschafften These der Sittenwidrigkeit dieser Tätigkeiten. ${ }^{1} 27$ Jahre später wurde das ProstG verabschiedet, trotz unterschiedlicher Ansichten der Parteien zur Prostitution. Während Bündnis 90/Die Grünen, SPD und PDS die Prostitution aufgrund der gewandelten Sitten und Sexualmoral mit anderen Berufen gleichstellen wollten, verwies die CDU/CSU darauf, dass die Prostitution nach ihrem Verständnis noch immer sittenwidrig sei und sie gerne mehr zum Schutz der Opfer von Menschenhandel getan hätte. ${ }^{2}$ Sie wollten Prostituierte nicht als selbstbestimmte erwachsene Menschen ansehen, sondern degradierten sie zu Opfern von Menschenhandel, leichtgläubig, unreif und schutzbedürftig. Helfen wollte man ihnen beim Ausstieg, aber sie nicht bei der Verbesserung ihrer rechtlichen und tatsächlichen Situation unterstützen. ${ }^{3}$

An dieser Sichtweise änderte sich auch nach der Veröffentlichung der drei Evaluationsgutachten zum ProstG im Jahre 2007, trotz einer Vielzahl darin unterbreiteter Vorschläge und Empfehlungen zur Verbesserung der Lage, nichts. ${ }^{4}$ Im Jahre 2010 begann das BMFSFJ ${ }^{5}$ drei Modellprojekte zum Ausstieg aus der Prostitution zu fördern. Für die Verbesserung der rechtlichen und tatsächlichen Situation wurde aber immer noch nichts unternommen. Aber es gab Tagungen zu diesem Bereich, auf denen Gewerberechtler die Nutzung ihrer Instrumente forderten, um Bordellbetreiber zu zivilisieren. ${ }^{6}$
Im Februar dieses Jahres entschloss sich der Bundesrat zu einer „Stärkeren Reglementierung des Betriebs von Prostitutionsstätten“ und sieht in diesem Zusammenhang auch eine gewerberechtliche Erlaubnispflicht vor. Aber inhaltlich erfasst dieser Gesetzesentwurf fast keine der real existierenden Stätten, da in der Praxis selbstständige Prostituierte tätig sind, dieser Entwurf sie aber (im Zweifel) zu unselbständigen Arbeitnehmerinnen definieren will. ${ }^{7}$ Wer gehofft hatte, endlich einen Vorschlag zur Verbesserung der Situation für Prostituierte und Betreiber zu sehen, wird enttäuscht. Dabei hätte dies ein guter Ansatz sein können, das Gewerberecht anwendbar zu machen und auf diese Weise für alle Einrichtungen, die Sexdienstleistungen anbieten, eine gewisse Rechts- und Planungssicherheit zu schaffen. Neben den Betreibern würden von einer präventiven und eben nicht strafrechtlichen Prüfung auch selbstständig in diesen Stätten arbeitende Prostituierte profitieren. Koppelte man etwa die für alle geltende Erlaubnispflicht an einen Sachkundenachweises, wie es ihn in fast allen anderen Gewerben bereits gibt, würden Betreiber, bei denen es sich zum Großteil um Quereinsteiger handelt, zumindest die nötige Steuer- und Rechtskenntnisse vermittelt, welche Rechtsverstöße aus Unkenntnis verhindern könnten. ${ }^{8}$

Liest man den Beschluss des Bundesrates, findet sich nichts, was die Situation für Betreiber oder Prostituierte verbessern könnte. Wie fast immer bei diesem Thema geht es stattdessen um Menschenhandelsopfer, Zwangsprostituierte und ausbeuterischen Zuhälter, die allenthalben mit Prostituierten und Betreibern in "einen Topf geworfen“ werden; ProstG hin oder her. Es scheint, dass sich die Ansichten seit 2002 nicht verändert haben. Die Einleitung zu dem Beschluss endet: 
„Aus Sicht der Bundesregierung bedarf es deshalb eines insgesamt breiteren Ansatzes der Reglementierung der Prostitution, der insbesondere konsequent die Bekämpfung von Menschenhandel, Zwangsprostitution und Minderjährigenprostitution integriert und auf einen größtmöglichen Schutz von Prostituierten vor Gewalt und Ausbeutung abzielt...."

Wieder geht es nicht um eine Verbesserung der Lage, sondern um eine Kriminalisierung dieser, was an den 7 Punkten, die laut Beschluss als Gesetzesinhalt aufgenommen werden sollen, deutlich wird.

\section{Punkt 1: Erlaubnispflicht für Prostitutionsstätten}

Die Erlaubnispflicht soll neben den Möglichkeiten der Befristung und Erteilung von Auflagen auch eine Versagung vorsehen, wenn „das Betriebskonzept erhebliche Nachteile oder Belästigungen für die Jugend, die Allgemeinheit oder die Umwelt befürchten lässt (beispielsweise weil eine Flatrate-Kalkulation vorgesehen ist) oder die Antragsteller wegen einschlägiger "Milieudelikte“ vorbestraft sind."

Dass die Flatrate-Bordelle explizit aufgeführt sind zeigt, dass diese wohl nicht in die aufgezählten Nachteile passen würden, sie aber den Ausschlag zum vorliegenden Beschluss gaben. Warum soll gerade diese Betriebsart eine Erlaubnisversagung erhalten? Von vielen Prostituierten werden solche Bordelle geschätzt, da sie hier keinen Eintritt zahlen müssen und einen festen Verdienst pro Abend einplanen können. ${ }^{9}$ Die Berichterstattung und die Initiativen zur Schließung der „Pussy-Clubs“ im Jahre 2009 sorgten aber offensichtlich dafür, dass nun eine gesetzliche Grundlage geschaffen werden soll, schnell gegen unliebsame Betriebsarten vorgehen zu können. ${ }^{10}$ Damit setzen sich Betreiber der Gefahr aus, dass ihre Erlaubnis auch entzogen wird, wenn sie gegen keinen der aufgeführten Nachteile verstoßen.

Die Formulierung „einschlägige Milieudelikte“ ist erstaunlich unbestimmt. Bis 2002 war die Förderung der Prostitution noch strafbar, wie verhält es sich mit solchen Vorstrafen? ${ }^{11}$ Was sind einschlägige Milieudelikte? Betreiber werden damit erneut kriminalisiert, sämtliche Straftatbestände können als Milieudelikte angesehen werden, unterstellt man eine grundsätzliche Kriminalitätsneigung.

Weiter sollen Mindestanforderungen unter hygienischen und sicherheitsrelevanten Aspekten festgelegt werden, was grundsätzlich zu begrüßen ist. Seit Jahren gibt es Forderungen nach Mindeststandards für Bordelle und Straßenstriche aus den Reihen der Prostituierten und Beratungseinrichtungen. ${ }^{12}$ Solche müssen aber zunächst erstellt werden, bevor sie an eine Erlaubnispflicht gekoppelt werden können, da es solche bisher, ebenso wenig wie eine Klassifizierung oder Eingruppierung der verschiedenen Bordellbetriebe, gibt.

\section{Punkt 2: Meldepflicht}

Prostitutionsstätten sollen einem engmaschigen System an Meldepflichten unterliegen.

Hierfür soll der Betreiber verpflichtet werden „bereits am Tag der Beschäftigungsaufnahme sämtliche relevanten Daten aller dort tätigen Personen auch der im weiteren Umfeld Beschäftigten, ... zu ... melden".

Darüber hinaus soll das Beschäftigungsende binnen Wochenfrist mitgeteilt werden, „... damit lückenlose und aktuelle Informationen über sämtliche Beschäftigten vorliegen und - sämtliche mit den Prostituierten geschlossenen Verträge (Beschäftigungsverträge, Mietverträge, Zusatzvereinbarungen usw.) vorliegen.

Das Prostitutionsgewerbe funktioniert aber anders. Es gibt eine extrem hohe Mobilität. Oftmals arbeiten Prostituierte nur wenige Tage an einem Ort und die Betreiber kennen nur das Pseudonym.
Eine Mitteilung aller relevanten Daten, noch am Tage der Beschäftigungsaufnahme, ist in der Praxis nahezu unmöglich. Oftmals entscheiden die Prostituierten spontan, ob sie arbeiten wollen oder nicht. Mit einer solchen Regelung sind Verstöße vorprogrammiert. Darüber hinaus müssen die Prostituierten ihre vollständigen Daten ständig anderen Betreibern mitteilen. Da viele ein Doppelleben führen, machen sie sich damit erpressbar und erst recht „abhängig von den Betreibern “. ${ }^{13}$ Aufgrund der hohen Flexibilität, was Einsatzort und Einsatzzeit angeht, arbeiten die meisten der Prostituierten selbstständig und nicht in abhängiger Beschäftigung. Sie wollen nicht weisungsgebunden sein und selbst entscheiden, was sie wann für welchen Betrag anbieten. Die Betreiber haben keine Möglichkeit, Prostituierte abzumahnen oder auf ihre Dienstleistungen Einfluss zu nehmen, da ihnen dies durch $₫ 3$ ProstG verwehrt ist, weshalb abhängige Beschäftigungen auch für sie nicht erstrebenswert sind. ${ }^{14}$

Darin liegt auch der Grund, dass bisher nahezu keine Arbeitsverträge in diesem Bereich geschlossen wurden.

\section{Punkte 3 und 4: Prävention sexuell übertragbarer Krankheiten und Sanktionsmöglichkeiten}

Bordellbetreiber sollen auf Risiken von ungeschütztem Geschlechtsverkehr hinweisen. In Punkt 3 heißt es dazu: „Der Betreiber muss auf die Kondompflicht deutlich sichtbar hinweisen und darf ungeschützten Geschlechtsverkehr nicht zulassen."

Diese Regelung ist auf den ersten Blick missverständlich. Angenommen werden könnte, dass falls eine Prostituierte ihre Dienstleistung ohne Kondom erbringt, im Wiederholungsfall dem Betreiber die Erlaubnis entzogen werden kann. ${ }^{15}$ Da die Einhaltung der Kondompflicht faktisch nicht lückenlos zu überwachen ist, böte sich bei dieser Gesetzeslage sogar ein Einfallstor für Konkurrenten, die so gezielt den Entzug der Erlaubnis des unerwünschten Kollegen erschleichen können. Gemeint ist aber wohl, dass der Bordellbetreiber alle in seinem Betrieb arbeitenden Prostituierten darauf hinweisen muss, dass er ungeschützten Geschlechtsverkehr in seinen Räumen nicht duldet und bei Zuwiderhandlung ggf. der entsprechenden Person Hausverbot erteilt. Damit käme er seiner Kondompflicht nach.

\section{Punkt 5: Vermutung abhängiger Beschäftigung und Präzisierung des Weisungsrechts}

„Bei Prostituierten, die ihre Dienstleistung innerhalb einer Betriebsstätte erbringen, soll grundsätzlich abhängige Beschäftigung angenommen werden, es sei denn die Deutsche Rentenversicherung stellt positiv fest, dass die Beschäftigung nicht sozialversicherungspflichtig ist. "Eine selbstständige Tätigkeit ist damit für Prostituierte nur noch auf dem Straßenstrich oder in Wohnungsbordellen möglich. Wenn in der Einleitung explizit darauf hingewiesen wird, dass man mit diesem Gesetz der Abhängigkeit zwischen Prostituierten und Betreibern entgegenwirken wollte, ist dies erstaunlich.

Eine grundsätzliche Annahme abhängiger Beschäftigung ist weder im Sinne der Prostituierten, noch im Sinne der Betreiber. ${ }^{16}$ Vielmehr ist dieser Punkt dadurch motiviert, dass Verfahren wegen eines möglichen Verstoßes gegen $\$ 266$ a StGB bzw. die Steuereintreibung für den Staat vereinfacht werden. Immerhin scheint die Problematik gesehen worden zu sein, bewirkte aber leider kein Umdenken. Im Beschluss heißt es: „Es ist dabei aber auch zu berücksichtigen, dass zu bohe Anforderungen an den Nachweis einer selbständigen Tätigkeit die ungewollte Folge haben können, dass Prostituierte, die selbstbestimmt arbeiten möchten, auf den Straßenstrich oder Prostitutions- 
formen außerhalb von Prostitutionsstätten ausweichen " und weiter: „Das in $\int 3$ des Prostitutionsgesetzes geregelte eingeschränkte Weisungsrecht für Betreiber von Prostitutionsstätten wird zur Stärkung der Weisungsfreiheit und der Vorbengung wirtschaftlicher oder persönlicher Abhängigkeit der Prostituierten präzisiert. "Wie dies erfolgen soll, obwohl eine abhängige Beschäftigung angenommen wird, die selbstverständlich zu Regelung der Arbeitskleidung, der Arbeitszeiten, der anzubietenden Dienstleistungen, der Preise etc. führen muss, wird nicht ausgeführt.

\section{Punkte 6 und 7: Änderung des Jugendschutzgesetzes und des Bundeszentralregistergesetzes}

Abschließend wird noch ein Anwesenheitsverbot Minderjähriger in Prostitutionsstätten durch Änderung des Jugendschutzgesetzes und eine Änderung des Bundeszentralregistergesetzes insofern, als dass die für die Erlaubnispflicht zuständigen Behörden uneingeschränkte Auskunftsmöglichkeiten über Eintragungen von Antragstellern erhalten sollen.

\section{Fazit}

Der Beschluss geht an der Problematik vorbei. Prostituierte und Betreiber sind weder „geborene“ Opfer noch Kriminelle. Das ProstG geht davon aus, dass es legale Möglichkeiten geben soll. Die permanente Vermischung der Gruppen ist daher ein grober Eingriff in das Selbstbestimmungsrecht derjenigen, die legal arbeiten wollen.

Dass das ProstG bisher nur unzureichend implementiert wurde, liegt daran, dass die Praxis nicht konstruktiv vorgeht und weder den Prostituierten noch den Betreibern zugesteht, dass sie sich wie andere Unternehmer nach dem Gewerberecht richten wollen. ${ }^{17}$ Noch immer wird die Anpassung der nicht-strafrechtlichen Rechtsgebiete an das Prost $G$ verweigert, es gibt weder klare steuer-, bau- und ordnungsrechtliche Regelungen noch akzeptiert man, dass diese Tätigkeit die Folge eigenverantwortlichen Handelns ist. Stattdessen werden Prostituierte und Betreiber als Opfer und Kriminelle dargestellt, denen man nicht das zutraut, was man anderen erwachsenen Menschen auch zugesteht, Risiken zu erkennen und ihre Folgen zu vermeiden. Solange sich diese Haltung nicht ändert, wird man das Thema Prostitution nur in einem Atemzug mit Menschenhandel ansprechen, keine Verbesserung der rechtlichen und wirtschaftlichen Lage der Prostituierten anstreben und Betreiber nicht zu transparenten Regelungen zwingen. Der Mythos des Rotlichtmilieus scheint sentimentale Bedürfnisse zu befriedigen. Seit einigen Jahren bemäntelt man diese Rückständigkeit auch noch feministisch und mit den ehrwürdigen Zielen des Opferschutzes.

Dr. Ina Hunecke ist Strafrechtswissenschaftlerin und hat sich auf das Thema der Regulierung der Prostitution spezialisiert.

\section{Fußnoten}

1 Hunecke, Das Prostitutionsgesetz und seine Umsetzung, Kovac 2011, S. 79.

2 Grenz/Lücke.-Dodillet, ,Verhandlungen im Zwielicht, Momente der Prostitution in Geschichte und Gegenwart“, Transcript Verlag 2006, S. 107, 108; Bt-Drs. 14/6781, Entschließungsantrag der CDU/CSU vom 3.7.2011., "Hierbei forderten sie, die rechtlichen Gestaltungen, welche die sittenwidrig begründeten Forderungen mit Mitteln der staatlichen Rechtsordnung zur Durchsetzung verhelfen sollten, auszusparen und besonderes Augenmerk auf die Opfer von Menschenhandel und Zwangsprostitution zu legen.“

3 Hunecke, S. 83. In seiner Begründung führte der Entschließungsantrag unter 4. aus, dass ein wichtiger Gesichtspunkt bei den Überlegungen die Verbesserung der Möglichkeiten für ausstiegswillige Prostituierte sei. Ebenfalls an dieser Stelle wurde ausgeführt, dass etwa die Hälfte der Prostituierten als Menschenhandelsopfer, Zwangsprostituierte oder Ausländer diese Tätigkeit illegal ausübten.

4 Gutachten: „Ausstieg aus der Prostitution“, Kriminalitätsbekämpfung und ProstG“ sowie Abschlussbericht „Auswirkungen des ProstG“ von SoFFiK und „Reglementierung von Prostitution“von Renzikowski.

5 Bundesministerium für Familie, Senioren, Frauen und Jugend.

6 Z.B. Kommunalakademie 2009, 2010 „Raus aus der Grauzone“.

7 Beschluss des Bundesrates vom 11.02.2011, Drs. 314/10. Darin bittet der Bundesrat die Bundesregierung eine gesetzliche Regelung für den Prostitutionsbereich mit folgenden Gesetzesinhalten zu erlassen.

8 Auskunft des Präsidenten des UEGD, Holger Rettig.

9 Offener Brief der Sexarbeiter des Pussy-Clubs (FN. 799, Diss. S. 248)

10 Darstellung der Entwicklungen und Veröffentlichungen zur Flaterate Debatte anläßlich der Prosteste gegen die Pussy-Clubs in Hunecke, S. 246-260.

11,180 a StGB wurde von „Förderung der Prostitution“ im Zuge des In Kraft treten des Prostitutionsgesetzes in „Ausbeutung von Prostituierten“ geändert. Bis 2002 war alles, was über eine Wohnungsgewährung hinaus ging strafbar.

12 Hunecke, S. 249, Offener Brief verschiedener Beratungsstellen aus Juli 2009. Darin beklagten sie, dass die Stellungnahmen der Betroffenen nicht gehört wurde und dass „, bis heute keine beruflichen Standards festgeschrieben und keine Instanzen, wie beispielsweise Berufsverbände geschaffen und unterstützt würden, die solche Standards entwickeln und ihre Einhaltung kontrollieren könnten.“

13 Hunecke, S. 208 f.

14 Schriftliche Stellungnahme des UEGD vom 2.4.2011.

15 Unter Punkt 4 „Sanktionsmöglichkeiten findet sich dann auch der entsprechende Passus: „... Verstöße gegen die in den Nummern 1 bis 3 genannten weiteren Pflichten stellen Ordnungswidrigkeiten dar und werden mit einem Bußgeld geahndet. Sie können zusätzliche Auflagen nach sich ziehen und im Wiederholungsfall bis hin zum Verlust der Betriebserlaubnis oder zur strafrechtlichen Sanktionierung führen."

16 „Colosseum Verfahren“, näheres in Hunecke, S. 208 f. Das Gericht führt in seinem Beschluss dezidiert aus, dass es sich bei der Art der Beschäftigung nicht um eine Abhängige handele. Als Gründe führte das Gericht an, dass die Sexarbeiter sich zwar aufgrund freiwilliger Entscheidung für bestimmte Tage im „Colosseum" anmelden und dann auch eine Anwesenheitspflicht bestünde, sie jedoch keine starren Stundenfristen einzuhalten hätten. Die Anwesenheitspflicht diene mehr der Organisation von Angebot und Nachfrage, es stehe den Sexarbeitern frei, etwas später zu kommen oder früher zu gehen. Die Leistungen müssten zwar im „Colosseum“ erbracht werden, daraus könne aber noch nicht geschlossen werden, dass ein abhängiges Beschäftigungsverhältnis vorliege. Zum einen könnten die Sexarbeiter außerhalb der Arbeitszeiten, ihrem Gewerbe frei an anderen Orten nachgehen, zum anderen zahlten die Sexarbeiter an den Tagen, an denen sie im „Colosseum“ arbeiteten, Eintritt.

17 Ausführlich hierzu Hunecke S. 333 ff.. 